\title{
Primate Prefrontal Cortex Evolution: Human Brains Are the Extreme of a Lateralized Ape Trend
}

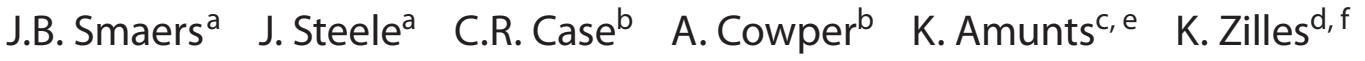 \\ ${ }^{a}$ AHRC Centre for the Evolution of Cultural Diversity, London, UK; ${ }^{b}$ Department of Anthropology, Miami University, \\ Oxford, Ohio, USA; ' Institute of Neuroscience and Medicine (INM)-1 and dINM-2, Research Centre Jülich, Jülich, \\ ${ }^{e}$ Clinic of Psychiatry and Psychotherapy, University Hospital Aachen, Aachen, and ${ }^{f}$ C. \& O. Vogt Institute of Brain \\ Research, University of Düsseldorf, Düsseldorf, Germany
}

\section{Key Words}

Prefrontal cortex $\cdot$ Neocortex $\cdot$ Lateralization · Asymmetry • Primate $\cdot$ Allometry

\begin{abstract}
The prefrontal cortex is commonly associated with cognitive capacities related to human uniqueness: purposeful actions towards higher-level goals, complex social information processing, introspection, and language. Comparative investigations of the prefrontal cortex may thus shed more light on the neural underpinnings of what makes us human. Using histological data from 19 anthropoid primate species (6 apes including humans and 13 monkeys), we investigate crossspecies relative size changes along the anterior (prefrontal) and posterior (motor) axes of the cytoarchitectonically defined frontal lobe in both hemispheres. Results reveal different scaling coefficients in the left versus right prefrontal hemisphere, suggest that the primary factor underlying the evolution of primate brain architecture is left hemispheric prefrontal hyperscaling, and indicate that humans are the extreme of a left prefrontal ape specialization in relative white to grey matter volume. These results demonstrate a neural adaptive shift distinguishing the ape from the monkey radiation possibly related to a cognitive grade shift between (great) apes and other primates.
\end{abstract}

Copyright ๑ 2011 S. Karger AG, Basel
(C) 2011 S. Karger AG, Basel

0006-8977/11/0772-0067\$38.00/0

Fax +4161306 1234

E-Mail karger@karger.ch

www.karger.com
Accessible online at:

www.karger.com/bbe

\section{Introduction}

The evolution of the prefrontal cortex in primates has captured the attention of neuroscientists for decades because of its purported role in human-like cognitive capacities such as coordinated purposeful behaviour towards reaching higher-level goals [Asplund et al., 2010; Miller and Cohen, 2001], high levels of social information processing [Adolphs, 2009], metacognitive introspective abilities [Fleming et al., 2010], and language. Early allometric studies indicated a disproportionate increase in the human prefrontal cortex compared to other primates [Blinkov and Glezer, 1968; Brodmann, 1912; Passingham, 1973] leading to suggestions that the prefrontal cortex is the neural basis of what makes humans unique [Deacon, 1997]. More recent studies investigating relative size expansion of prefrontal subregions suggest that prefrontal expansion may be characterized by more than the predicted expansion in particular prefrontal regions (e.g. area BA10 and Broca's area in humans) rather than a general expansion of the prefrontal cortex as a whole [Schenker et al., 2010; Semendeferi et al., 2001]. Research looking at the cytoarchitectonic organization of prefrontal areas such as Broca's area suggests prefrontal homologies linking human to ape and even monkey brains [Petrides, 2005; Petrides et al., 2005; Schenker et al., 2008; Taglialatela et al., 2008]. These studies call into question whether

Dr. Jeroen Smaers

AHRC Centre for the Evolution of Cultural Diversity

31-34 Gordon Square

London WC1H 0PY (UK)

Tel. +44 122376 4719,E-Mail j.smaers@ucl.ac.uk 
the uniqueness of the human brain is solely a matter of the evolution of new areas and further emphasize the importance of investigating putative patterns of change in relative prefrontal size in order to better understand whether relative size changes of the prefrontal cortex are a significant factor in the evolution of primate brain organization and which aspects of human prefrontal size changes stand out.

Suggestions towards human phyletic uniqueness of the prefrontal cortex have mainly come from allometric studies. These studies identify quantitative structural changes in a brain structure (e.g. relative size, cortical folding, white to grey matter ratio) relative to the predicted changes observed across the primate order. More specifically, allometric studies investigate the extent to which quantitative structural changes in particular structures scale disproportionately (non-isometrically or allometrically) compared to other structures over the course of millions of years of evolution across many species. Although allometric deviations do not allow direct functional inferences [Passingham, 2002], allometric trends are crucial because they affect size-dependent cytoarchitectonic features such as cell morphology, neural/glial ratio, lamination and myelination. Moreover, there is increasing evidence in humans [Carreiras et al., 2009; Draganski et al., 2004; Fleming et al., 2010; Scholz et al., 2009] and extensive longstanding evidence across the animal kingdom [DeVoogd et al., 1993; Huber et al., 1997; Iwaniuk and Wylie, 2007; Smith et al., 2010] that quantitative changes in brain anatomy correlate to behavioural capacity. An allometrically observed deviation in quantitative structural changes of a particular structure in a particular species may therefore contribute to explaining deviated (or specialized) function.

Studies investigating whether the relative size of the human prefrontal cortex deviates from what is predicted based on the primate pattern (i.e. whether the human prefrontal cortex is indeed relatively larger than predicted for a primate of our brain size) have come to different conclusions: some authors have suggested that humans have a significantly larger prefrontal cortex than predicted [Blinkov and Glezer, 1968; Brodmann, 1912; Deacon, 1997; McBride et al., 1999; Passingham, 1973; Schoenemann et al., 2005], while others suggest human frontal and prefrontal cortex is not larger than predicted [Bush and Allman, 2004; Holloway, 2002; Jerison, 1997, 2007; Semendeferi et al., 2002; Uylings and Van Eden, 1990] and human prefrontal evolution may be characterized by more than the predicted expansion in particular prefrontal regions rather than a general expansion of the prefron- tal cortex as a whole [Semendeferi et al., 2001]. This contention is largely due to difficulties in mapping cy toarchitectonic landmarks of the prefrontal cortex across primates [Semendeferi et al., 2002; Sherwood et al., 2005], resulting in different anatomical methods and different definitions of frontal and prefrontal cortex. Currently, there is no common agreement on whether the human prefrontal cortex as a whole is, in fact, disproportionately larger compared to that in other primates.

Besides questions pertaining to relative size, questions about prefrontal asymmetry have been largely ignored in comparative allometric studies despite suggestions of prefrontal functional asymmetries in humans [Berkman and Lieberman, 2010; Corbetta and Shulman, 2002], the demonstration of structural asymmetries in human prefrontal areas such as Broca's area [Amunts et al., 1999; Uylings et al., 2006] and the observation of frontal neuroanatomical asymmetries related to the production of lateralized communicative behaviour in chimpanzees [Taglialatela et al., 2006]. Considering the functional and structural prefrontal asymmetries observed in humans, the prefrontal cytoarchitectonic homologies in prefrontal areas such as Broca's area between humans and other primates and the cortical asymmetries found in chimpanzees [Hopkins, 2007; Hopkins et al., 2007a-c; Hopkins et al., 2008] one may predict prefrontal structural asymmetries also to occur in other primates. We investigate here allometric trends in prefrontal white matter, grey matter and white + grey matter (i.e. overall volume) in 19 primate species (fig. 1) in both left and right hemispheres. We use cytoarchitectonic criteria to delineate the frontal lobe and subsequently use a bootstrap-like procedure to investigate allometric trends in the anterior (prefrontal) and posterior (motor) areas of the frontal lobe. Through incorporating data on both hemispheres, we investigate putative prefrontal hemispheric structural asymmetries.

\section{Materials and Methods}

Brain Data and Delineation Procedure

Brain Data

We examined brains from 19 anthropoid species (including humans). For all non-human primate species 1 individual was analyzed, for humans 8 individuals were analyzed. All brain data is housed at the C. \& O. Vogt Institute for Brain Research (Heinrich Heine University, Düsseldorf, Germany). This collection consists of serial, Nissl-stained brain sections. Because previous research has suggested sexual dimorphism in primate brain structure evolution is expected to occur [Lindenfors, 2005; Lindenfors et al., 2007], we focus on males to avoid the possible confounding effect of putative sex differences. Of the 18 non-human 
species in our sample, only 4 are female: Pan paniscus, Nasalis larvatus, Procolobus badius, and Lagothrix lagotricha. Of the humans, 3 are female: 54491, 5694, and 6895.

Frontal Delineation Procedure

The frontal lobe is defined as the part of the neocortex anterior to the boundary between the primary motor cortex (area 4) and the somatosensory cortex (area 3). The border between area 4 and area 3 is defined based on six cytoarchitectonical changes between area 4 and area 3 [Bush and Allman, 2004; Sherwood et al., 2004]: cell shape (pyramidal and elongated Betz cells vs. absence of Betz cells); cell density (low vs. high); cell size (large vs. small); granular layer IV (absent vs. present); border between white and grey matter (diffuse vs. sharp), and lamination (diffuse vs. sharp). The cortical boundary between area 4 and area 3 was mapped by J.B.S. in non-human primates and taken from the Jülich/Düsseldorf brain atlas (data from Geyer et al. [1999] and Matelli et al. [2004]) in human individuals. The boundaries between cortical white and grey matter and between cortical white matter and non-cortical structures were mapped by J.B.S. in all individuals. For all individuals, frontal lobe volume was computed based on an analysis of 20 equidistant sections (for more details on the delineation and volume estimation procedures used, see Smaers et al. [2010]). Frontal lobe and total brain volumes for all individuals are presented in online supplementary table 1 (www. karger.com/doi/10.1159/000323671).

Prefrontal Delineation Procedure

For each brain, all delineation procedures were applied separately within each hemisphere. Considering the difficulties in mapping cytoarchitectonic landmarks of the prefrontal cortex across a large sample of primate species, we applied a bootstraplike approach based on the cytoarchitectonic delineation of the frontal lobe (for which landmarks are commonly agreed upon). The frontal lobe was subdivided into an anterior and posterior end in order to investigate those areas in which the prefrontal and frontal motor areas are located. The subdivision between anterior and posterior frontal lobe was attained by separately analyzing the most anterior sections (first 10 out of a total of 20 sections from the anterior to the posterior border of the frontal lobe) from the most posterior sections (first 10 out of a total of 20 sections from posterior to anterior border). We then calculated volumes cumulatively along both the anterior and posterior ends of the frontal lobe (volume up to the 1st section, up to the 2nd section, up to the 10th section), providing a cumulative distribution of volumes along the anterior and posterior ends of the frontal lobe. Cumulative volumes along the first three sections were not considered to avoid volumetric estimations based on too few sections (for both the anterior frontal and posterior frontal analyses). To investigate detailed allometric patterns along both the anterior and posterior ends of the frontal lobe, we computed (phylogenetic general linear model, PGLM) regressions at each section interval. For example, when investigating the relative volume along the anterior (prefrontal) end of the frontal lobe, we computed the cumulative volume along the anterior end of the frontal lobe and regressed the cumulative volume at each section interval (not including the first three) to 'rest of brain' volume (defined as total brain volume minus left frontal lobe volume for left anterior frontal and minus right frontal volume for right anterior frontal). This provides us with 7 regressions allowing a detailed investigation of

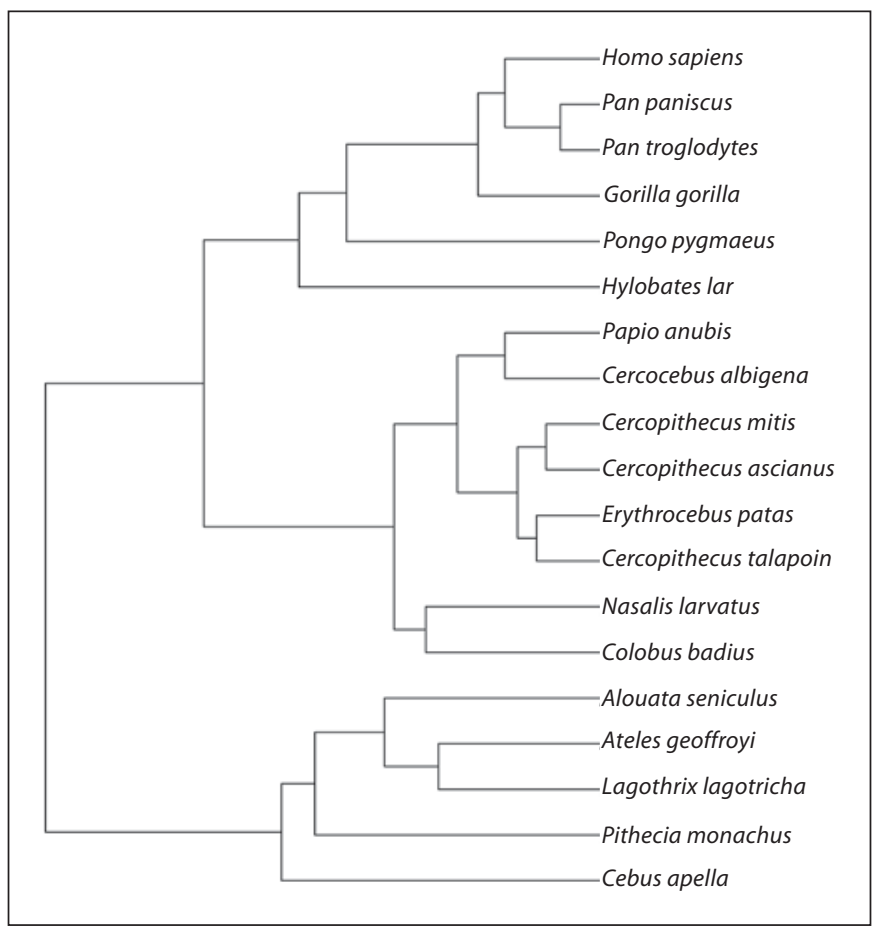

Fig. 1. Phylogenetic tree of species incorporated into the analyses.

the allometric trends of prefrontal volume to rest of brain at different positions along the anterior end of the frontal lobe. The same approach, with necessary changes, was applied for the posterior areas of the frontal lobe and the relative volume of the frontal motor areas. This procedure does not provide a single volumetric measure of either prefrontal or frontal motor areas, but investigates allometric trends at different positions along the anterior and posterior ends of the frontal lobe, providing a detailed overview of the volumetric evolutionary trends along those frontal areas where the prefrontal and frontal motor areas are located. Volumes of white and grey matter in both hemispheres up to the 5 th section of the anterior frontal are presented in online supplementary table 2 (www.karger.com/doi/10.1159/000323671).

\section{Statistical Procedure}

\section{Phylogenetic Controls}

Comparative phylogenetic methods are tools to deduce evolutionary trends from observed extant species data based on their phylogenetic relatedness [Harvey and Pagel, 1991]. Data were analyzed using the PGLM procedure as described by Gage and Freckleton [2003] and analyzed using the APE [Paradis et al., 2004] package in $R$ [R Development Core Team, 2010]. PGLM is equivalent to the phylogenetic generalized least squares approach [Martins and Hansen, 1997]. PGLM uses a maximum likelihood framework to estimate a parameter lambda, indicating the degree of phylogenetic dependence [Freckleton et al., 2002; Pagel, 1999]. Lambda varies between unity and zero where unity indicates that traits co-vary along the phylogeny according to their degree of shared ancestry (i.e. according to a Brownian motion model of trait evolution [Freckleton et al., 2002]) and zero indicates traits 
Table 1. Slopes and intercepts of PGLM regressions of overall volume (white + grey matter), white matter volume and grey matter volume to rest of brain volume for subsequent intervals (representing cumulative volume) along the anterior axis of the frontal lobe

\begin{tabular}{|c|c|c|c|c|}
\hline & \multicolumn{2}{|c|}{ Left hemisphere } & \multicolumn{2}{|c|}{ Right hemisphere } \\
\hline & slope & $95 \% \mathrm{CI}$ & slope & $95 \% \mathrm{CI}$ \\
\hline \multicolumn{5}{|c|}{ Prefrontal overall volume } \\
\hline Interval 4 & 1.35 & $1.20-1.49$ & 1.02 & $0.84-1.19$ \\
\hline Interval 5 & 1.35 & $1.21-1.49$ & 1.05 & $0.90-1.20$ \\
\hline Interval 6 & 1.33 & $1.20-1.45$ & 1.07 & $0.92-1.21$ \\
\hline Interval 7 & 1.29 & $1.17-1.41$ & 1.07 & $0.93-1.20$ \\
\hline Interval 8 & 1.23 & $1.12-1.34$ & 1.04 & $0.92-1.17$ \\
\hline Interval 9 & 1.20 & $1.09-1.31$ & 1.04 & $0.92-1.16$ \\
\hline Interval 10 & 1.16 & $1.06-1.26$ & 1.06 & $0.96-1.16$ \\
\hline \multicolumn{5}{|c|}{ Prefrontal white matter volume } \\
\hline Interval 4 & 1.77 & $1.48-2.05$ & 1.25 & $1.02-1.49$ \\
\hline Interval 5 & 1.70 & $1.45-1.95$ & 1.29 & $1.09-1.49$ \\
\hline Interval 6 & 1.62 & $1.39-1.84$ & 1.28 & $1.09-1.47$ \\
\hline Interval 7 & 1.54 & $1.33-1.75$ & 1.24 & $1.05-1.43$ \\
\hline Interval 8 & 1.43 & $1.23-1.64$ & 1.19 & $1.00-1.38$ \\
\hline Interval 9 & 1.38 & $1.19-1.57$ & 1.18 & $1.02-1.35$ \\
\hline Interval 10 & 1.34 & $1.17-1.52$ & 1.21 & $1.06-1.35$ \\
\hline
\end{tabular}

\begin{tabular}{lcccc}
\hline Prefrontal grey matter & volume & & \\
Interval 4 & 1.29 & $1.16-1.43$ & 0.97 & $0.81-1.14$ \\
Interval 5 & 1.29 & $1.16-1.43$ & 1.00 & $0.85-1.14$ \\
Interval 6 & 1.26 & $1.14-1.39$ & 1.02 & $0.88-1.15$ \\
Interval 7 & 1.23 & $1.11-1.35$ & 1.02 & $0.89-1.15$ \\
Interval 8 & 1.17 & $1.07-1.28$ & 1.00 & $0.87-1.12$ \\
Interval 9 & 1.14 & $1.04-1.24$ & 0.99 & $0.87-1.11$ \\
Interval 10 & 1.10 & $1.00-1.20$ & 1.00 & $0.90-1.10$ \\
\hline Posterior frontal overall volume & & \\
Interval 4 & 0.99 & $0.89-1.09$ & 1.04 & $0.88-1.19$ \\
Interval 5 & 1.03 & $0.94-1.13$ & 1.09 & $0.94-1.23$ \\
Interval 6 & 1.06 & $0.96-1.16$ & 1.10 & $0.97-1.23$ \\
Interval 7 & 1.07 & $0.97-1.18$ & 1.11 & $0.99-1.23$ \\
Interval 8 & 1.07 & $0.97-1.17$ & 1.10 & $0.99-1.22$ \\
Interval 9 & 1.06 & $0.95-1.16$ & 1.10 & $0.99-1.21$ \\
Interval 10 & 1.06 & $0.96-1.15$ & 1.10 & $1.00-1.20$ \\
\hline \multicolumn{7}{c}{ Intercept } & $95 \% \mathrm{CI}$ & \multicolumn{2}{c}{ Intercept $95 \% \mathrm{CI}$} \\
\hline
\end{tabular}

Prefrontal overall volume

$\begin{array}{lllll}\text { Interval 4 } & -6.40 & -7.07 \text { to }-5.74 & -4.76 & -5.56 \text { to }-3.96 \\ \text { Interval 5 } & -5.94 & -6.59 \text { to }-5.30 & -4.44 & -5.14 \text { to }-3.74 \\ \text { Interval 6 } & -5.44 & -6.04 \text { to }-4.85 & -4.12 & -4.78 \text { to }-3.46 \\ \text { Interval 7 } & -4.95 & -5.51 \text { to }-4.38 & -3.80 & -4.42 \text { to }-3.17 \\ \text { Interval 8 } & -4.40 & -4.92 \text { to }-3.88 & -3.41 & -4.00 \text { to }-2.82 \\ \text { Interval 9 } & -4.00 & -4.50 \text { to }-3.51 & -3.19 & -3.75 \text { to }-2.62 \\ \text { Interval 10 } & -3.62 & -4.10 \text { to }-3.14 & -3.11 & -3.57 \text { to }-2.65\end{array}$

Posterior frontal overall volume

\begin{tabular}{lllll} 
Interval 4 & -3.98 & -4.42 to -3.53 & -4.20 & -4.94 to -3.47 \\
Interval 5 & -3.86 & -4.31 to -3.41 & -4.11 & -4.78 to -3.44 \\
Interval 6 & -3.71 & -4.18 to -3.25 & -3.93 & -4.54 to -3.32 \\
Interval 7 & -3.58 & -4.07 to -3.10 & -3.75 & -4.32 to -3.18 \\
Interval 8 & -3.36 & -3.83 to -2.88 & -3.51 & -4.05 to -2.97 \\
Interval 9 & -3.11 & -3.59 to -2.64 & -3.31 & -3.82 to -2.80 \\
Interval 10 & -2.97 & -3.41 to -2.53 & -3.15 & -3.61 to -2.70 \\
\hline
\end{tabular}

co-vary independently of their degree of shared ancestry. PGLM obtains estimates of regression slopes incorporating the degree of phylogenetic dependence (between unity and zero) by reference to an internal matrix of expected covariance based on the maximum likelihood estimate of lambda. The phylogeny was taken from Smith and Cheverud [2002].

\section{Regression Procedure}

To estimate whether humans are aligned with the general anthropoid pattern, scaling coefficients, residuals and 95\% confidence intervals were calculated based on non-human anthropoid data. Human residuals were subsequently computed using the non-human scaling coefficients. This procedure provides a robust estimate that allows one to evaluate whether or not humans are aligned with other anthropoid primates in terms of quantitative structural changes along the (anterior and posterior) frontal lobe. Furthermore, all regression procedures were performed separately for each hemisphere. A disproportionate increase in relative volume in the right hemisphere must therefore be interpreted as an increase relative to the right hemisphere in other species and not as an increase relative to the left hemisphere. Following this procedure, any differences in relative volume of left versus right hemisphere are evaluated based on differences in allometric scaling coefficients (scaling coefficients of left hemisphere across species vs. scaling coefficients of right hemisphere across species). This procedure minimizes the potentially confounding effect of minor differences in section inclination relative to the longitudinal axis across species inherent to the histological process and allows estimating robust allometric differences between hemispheres revealing lateralization.

\section{Results}

\section{The Anthropoid Pattern: Lateralization of the Prefrontal Cortex}

The intercepts of the regression of prefrontal volume to rest of brain volume are consistently smaller in the left hemisphere compared to the right, while the slopes are consistently higher (table 1). These results apply for analyses involving overall prefrontal volume (white + grey matter) and for analyses of prefrontal white and grey matter separately (table 1). These results suggest smallerbrained anthropoids have relatively smaller left prefrontal cortex, but the relative size of the left prefrontal increases disproportionately as total brain size increases to the point where the left prefrontal is predicted to be larger in species for which rest of brain volume (total brain volume minus frontal lobe volume) is more than $150 \mathrm{ml}$ (fig. 2). Observed values align with this prediction in 12 out of 13 monkeys and 5 out of 6 apes: species for which rest of brain volume is larger than $150 \mathrm{ml}$ (baboon and great apes) indicate prefrontal volumes (up until the 9th anterior frontal interval) to be larger in the left compared to the right, and species with rest of brain volumes small- 
Fig. 2. PGLM regression of prefrontal volume in the left (full line) and right (dashed line) hemispheres at the 5 th cumulative interval along the anterior axis of the frontal lobe. Data points represent left hemispheric data.

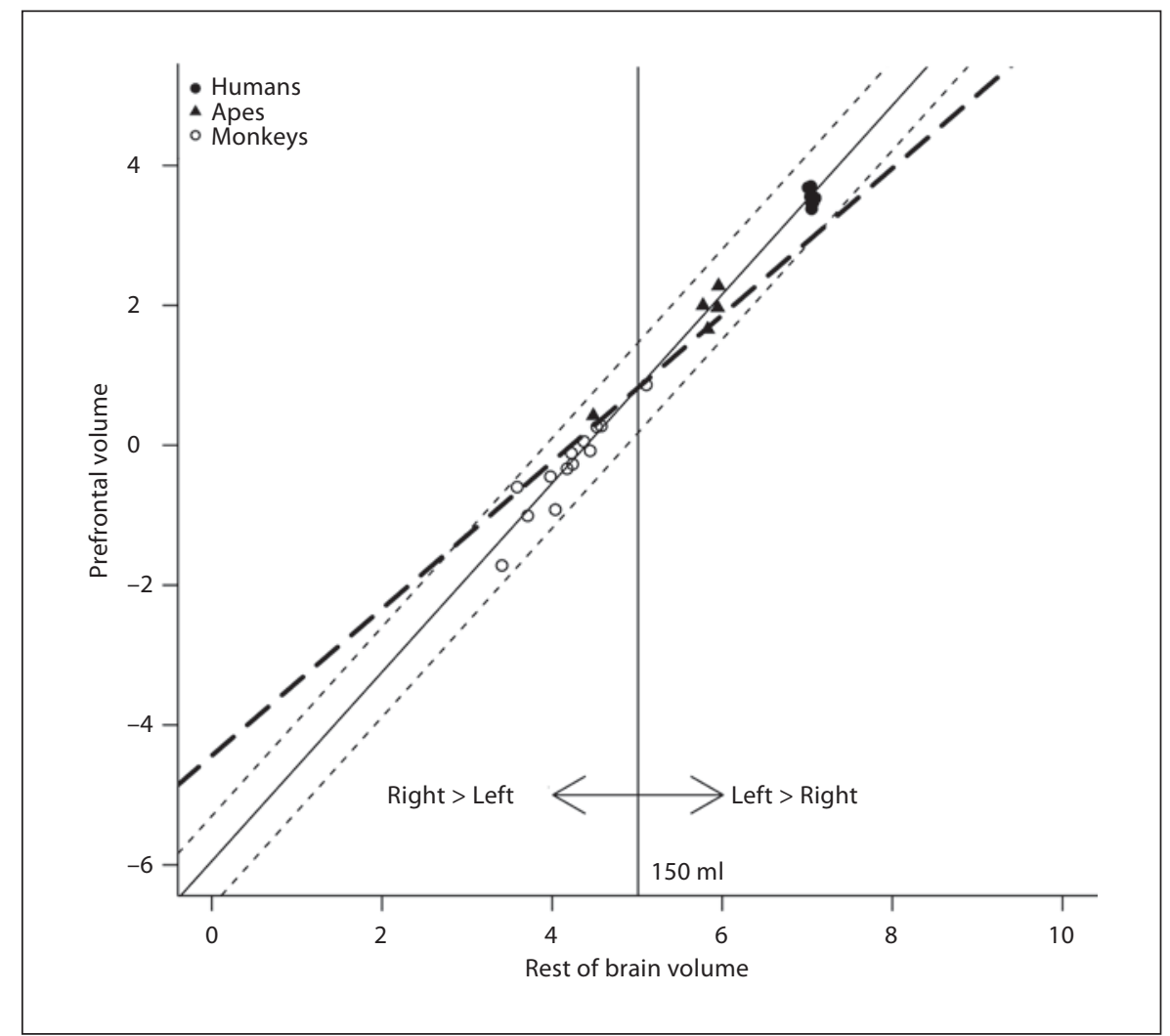

er than $150 \mathrm{ml}$ indicate prefrontal volumes in the left to be smaller than in the right. One ape and one monkey did not align with this general trend: in humans (rest of brain volume $>150 \mathrm{ml}$ ), volume along the anterior frontal axis is on average $2.45 \%$ larger in the right compared to the left; in Erythrocebus patas (rest of brain volume $<150 \mathrm{ml}$ ), the left is on average $0.04 \%$ larger than the right.

\section{Ape Uniqueness}

Along the anterior axis of the frontal lobe in the left hemisphere, residuals of the regression of prefrontal white to grey matter volume are elevated in apes and humans compared to monkeys (fig. 3a). Moreover, apes indicate a similar trend to humans, although human values are consistently around 1 residual higher and fall outside of the predicted non-human anthropoid pattern (fig. 3a, 4). In the prefrontal area of the right hemisphere, the ape (excluding chimpanzee, see below) and monkey trends are indistinguishable, but the human residuals are consistently higher than predicted (although less so than for the left hemisphere; fig. 3b). Residuals of white to grey matter volume along the posterior axis of the frontal lobe (frontal motor areas) indicate identical ape (excluding chimpanzee) and monkey patterns in both hemispheres (fig. 3c, d). In the left hemisphere of the posterior frontal lobe, human residuals are significantly higher than predicted (i.e. outside the $95 \%$ confidence interval; fig. 3c), but in the right hemisphere this is not the case as human residuals overlap with both apes and monkeys (fig. 3d).

\section{Human (and Chimpanzee) Uniqueness}

Human prefrontal volumes relative to rest of brain volume are in line with the non-human anthropoid pattern for both hemispheres and for white matter, grey matter and overall volumes. Figure 5 shows the PGLM regression of left prefrontal cortex overall volume (fig. 5a), and prefrontal white (fig. 5b) and grey matter (fig. 5c) volume to rest of brain volume for section interval 5 (similar results were found for all other section intervals along the anterior axis of the frontal lobe; see table 1), indicating that humans fall well within what is predicted based on the non-human anthropoid pattern. Humans indicate significantly more white to grey matter compared to the non-human anthropoid pattern in the 


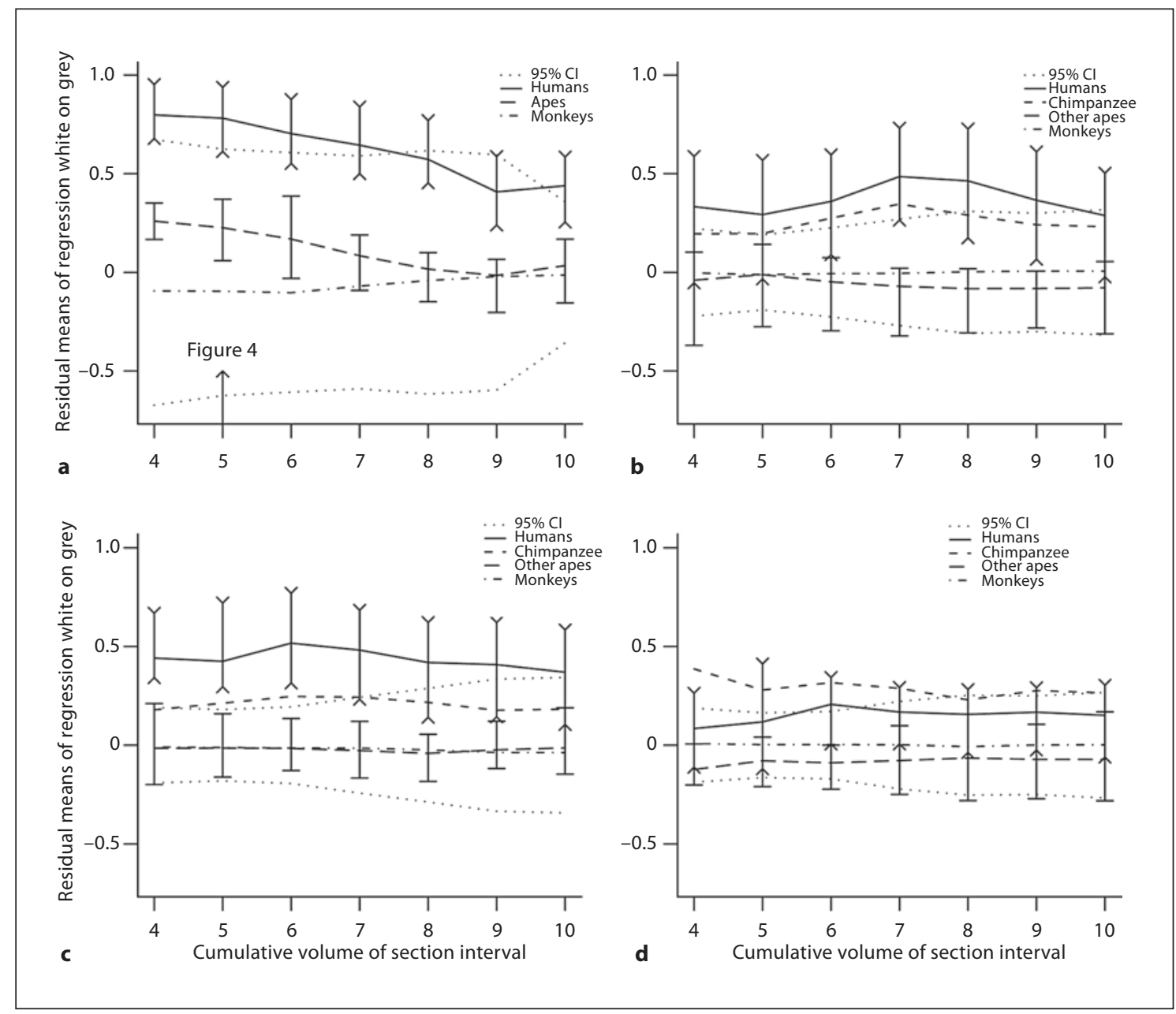

Fig. 3. Residual means of PGLM regressions of white matter volume on grey matter volume at subsequent cumulative intervals along the anterior $(\mathbf{a}, \mathbf{b})$ and posterior axes $(\mathbf{c}, \mathbf{d})$ of the frontal lobe in the left $(\mathbf{a}, \mathbf{c})$ and right hemisphere (b, d). Means were taken for humans, apes, and monkeys. Chimpanzee values are indicated separately in $\mathbf{c}, \mathbf{b}, \mathbf{d}$. Variation around the mean represents maxima and minima within each group.

left (fig. 3a) and right (fig. 3b) prefrontal area (with the left trend more pronounced) and in the left posterior frontal area (fig. 3c). This trend is most significant in the left hemisphere of both prefrontal and posterior frontal. In the most anterior intervals of the left prefrontal area (fig. 3a) and the most posterior intervals of the left frontal area (fig. 3c), no human individual lies below the upper 95\% confidence interval. Chimpanzee (Pan troglodytes) values fall in line with the ape trend for the left and right prefrontal (fig. 3a, b), lie between those of other non-human apes and humans for the right prefrontal (fig. 3b) and left posterior frontal (fig. 3c), and approximate the maximum of the human values in the right posterior frontal (fig. 3d).

\section{Discussion}

Despite suggestions of human prefrontal functional [Berkman and Lieberman, 2010; Corbetta and Shulman, 2002] and structural [Hugdahl and Westerhausen, 2010; Uylings et al., 2006; Zilles et al., 1996] asymmetry, evidence for prefrontal cytoarchitectonic homologies between humans and non-human primates [Petrides, 2005; Petrides et al., 2005; Schenker et al., 2008], and the importance of the prefrontal cortex for the evolution of human-like cognitive capacities [Deacon, 1997], there has been only little comparative investigation into the evolution of prefrontal structural asymmetries across primates [Falk et al., 1990; Heilbroner and Holloway, 1989; Schen- 
Fig. 4. PGLM regression of prefrontal white matter volume to grey matter volume in the left hemisphere at the 5 th cumulative interval along the anterior axis of the frontal lobe.

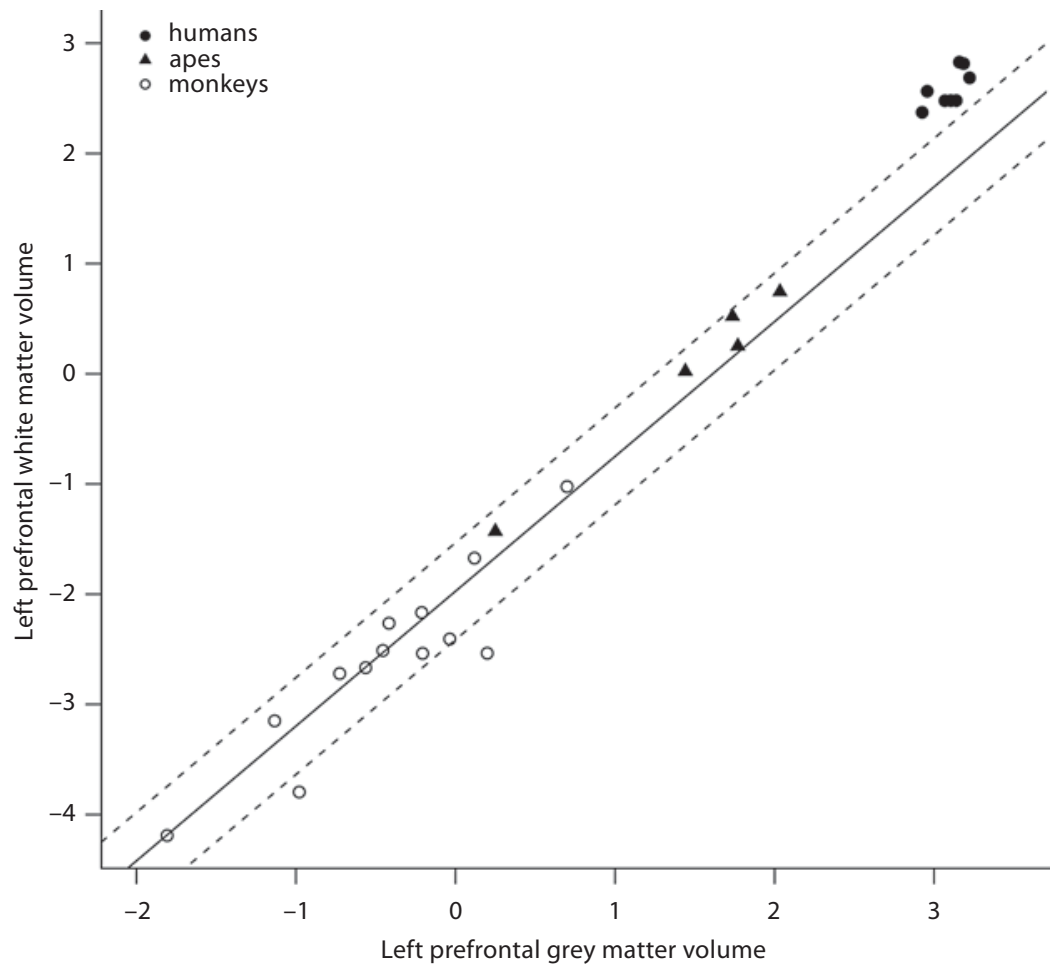

Left prefrontal grey matter volume

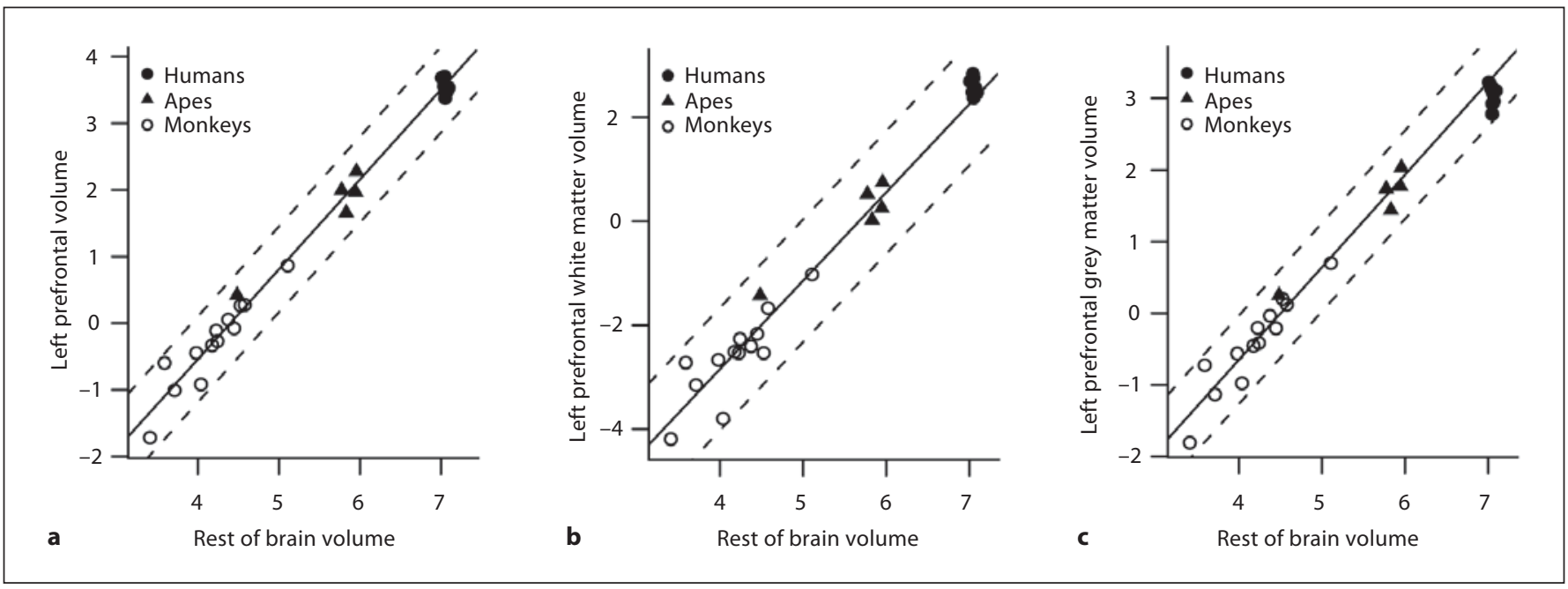

Fig. 5. PGLM regression of overall prefrontal (white + grey matter; a), prefrontal white matter (b) and prefrontal grey matter volume (c) to rest of brain volume in the left hemisphere at the 5 th cumulative interval along the anterior axis of the frontal lobe. 
ker et al., 2008; Sherwood et al., 2003]. Moreover, investigations into whether the human prefrontal cortex is exceptionally enlarged compared to other primates have been laden with contention because of difficulties in mapping prefrontal cytoarchitectonic landmarks across primates [Semendeferi et al., 2002; Sherwood et al., 2005], causing different authors to suggest different definitions of frontal and prefrontal cortex, resulting in different conclusions.

Schoenemann et al. [2005] used gross anatomical criteria (everything anterior to the corpus callosum) to define the prefrontal area and concluded that human prefrontal cortex volume and particularly prefrontal white matter volume are exceptionally enlarged, suggesting a more than predicted increase in human prefrontal neural connectivity. The gross anatomical criteria of Schoenemann et al. [2005] to define the prefrontal area are, however, by some considered problematic and may cast doubts on their conclusions [Sherwood et al., 2005]. We use cytoarchitectonic criteria to define the frontal lobe (for which landmarks are commonly agreed upon) and subsequently use a bootstrap approach to investigate volumetric changes along the anterior and posterior ends of the frontal lobe, employing the less problematic assumption that allometric trends along the anterior part of the frontal lobe accurately reflect those in the prefrontal cortex and allometric trends along the posterior part of the frontal lobe reflect those in frontal motor areas. Moreover, our approach includes separate analyses for each hemisphere, allowing inferring whether prefrontal cortex evolution is lateralized across primates.

Our results suggest that relative prefrontal volume is not exceptionally enlarged in humans at any part along the anterior axis of the frontal lobe (for both white matter, grey matter, and white + grey matter). Additionally, our results indicate a robust lateralization in the evolution of prefrontal volume. In anthropoid primates, smallerbrained species have a significantly smaller left prefrontal cortex and as brains enlarge, the left prefrontal cortex enlarges disproportionately more than the right. This trend applies for white matter, grey matter and overall volume (white + grey matter; table 1). For overall volume, $150 \mathrm{ml}$ is predicted as the rest of brain volume (defined as total brain volume minus left frontal lobe volume for left prefrontal and minus right frontal lobe volume for right prefrontal) at which the switch between smaller left than right and larger left than right occurs (fig. 2). Observed values confirm this prediction in 12 out of 13 monkeys and 5 out of 6 apes. The exception for the ape trend are humans who indicate a relatively higher increase in right prefrontal relative volume (human residuals are the highest in a regression of right prefrontal volume to rest of brain volume, but do not exceed the $95 \%$ confidence interval), resulting in minimal overall prefrontal volumetric lateralization in humans. The different allometric trends observed in the left and right hemispheres across primates are also observed when estimating the scaling coefficients based only on monkey data (slope left: 1.35; slope right: 0.91; intercept left: -5.99 ; intercept right: -3.87 ; 'switch' at $113 \mathrm{ml}$ of rest of brain volume). In other words, even without incorporating data on larger-brained species such as great apes, the monkey pattern correctly predicts the volumetric lateralization in larger-brained great apes (despite the fact that monkeys have the opposite volumetric lateralization).

Apes further distinguish themselves from monkeys in that they have evolved a distinct increase in relative white to grey matter volume in left (but not right!) prefrontal areas (fig. 3a, b). Humans follow this ape trend but to a degree significantly deviating from primate allometry (fig. 3a, 4), aligning with suggestions of a human specialization in prefrontal white matter [Schoenemann et al., 2005] and asymmetry of human white matter pathways [Catani et al., 2010]. Chimpanzees exhibit significantly more relative white to grey matter volume compared to other apes in the right prefrontal and left and right posterior frontal (fig $3 \mathrm{~b}-\mathrm{d}$ ). Increased cortical white matter in chimpanzees is supported by previous observations [Schenker et al., 2005] and recent work on the (extended) ontogenetic developmental growth of the prefrontal cortex in chimpanzees [Sakai et al., in press] provides a possible biological explanation for the here observed increased prefrontal white to grey matter volume, separating chimpanzees from other non-human apes. Increased white matter volume relative to grey matter volume is commonly interpreted as reflecting connectivity requirements related to increased neocortical size [Kaas, 2006], causing white matter to hyperscale to grey matter [Zhang and Sejnowski, 2000]. An increase in relative white to grey matter volume that is significantly higher than the predicted hyperscaling of white to grey matter volume may reflect a non-size-dependent increase in structural connectivity and/or increase in processing speed related to more integrated cognitive capacities.

The finding that, when compared to monkeys, apes (and especially humans) demonstrate a disproportionate enlargement of white relative to grey matter volume in the left (but not right) prefrontal, together with the observed volumetric lateralization favouring increased left to right prefrontal volume in great apes compared to 
monkeys, reflects a structural lateralization distinguishing the ape from the monkey radiation. This structural lateralization in (great) apes (volumetric lateralization in great apes and white to grey matter lateralization in apes) may reflect a functional lateralization underlying a cognitive grade shift between (great) apes and other primates. Inferring the behavioural implications of such a distinction remains difficult, although assuming a structural/functional correlation, possible pathways can be inferred from studies indicating prefrontal function and prefrontal functional asymmetries.

Language has long been associated with prefrontal function, and left hemisphere dominance in language processing is broadly supported. Moreover, recent research has shown that this hemispheric dominance for language in humans translates into structural asymmetry in prefrontal areas such as Broca's area [Amunts et al., 1999; Uylings et al., 2006]. Our results suggest left prefrontal structural specialization is not unique to humans, demonstrating consistent prefrontal structural lateralization in the ape clade. Assuming left prefrontal specialization for increased structural connectivity in humans can plausibly be associated with the evolution of language, our results support the notion that humans have built their language capacities on a pre-existing neural platform present in apes. This suggestion meshes well with research linking complex social information processing to prefrontal cortex evolution [Dunbar and Shultz, 2007] and suggestions that language evolution is a special case of the evolution of social information processing [Dunbar, 1996].

Human handedness has been found to be important in the context of tool use production [Marchant et al., 1995], and to be related to structural asymmetry in the motor cortex [Amunts et al., 1998, 2000]. Prefrontal regions have also been associated with tool use as part of a neural circuit for complex tool use [Johnson-Frey, 2004] and as part of general problem-solving abilities [Gray and Thompson, 2004]. Our result that humans show significantly more relative white to grey matter volume in the left compared to the right posterior frontal area may be interpreted in terms of structural asymmetries of the human motor cortex involved in handedness. Handedness and associated neuroanatomical asymmetries have also been suggested to be present in chimpanzees [Dadda et al., 2008; Hopkins et al. 2007a-c]. Our results for chimpanzees indicate increased relative white to grey matter volume compared to other apes in the posterior frontal area of both left and right hemispheres (fig. 3c, d). Assuming a link between structural connectivity and func- tional specialization in the motor system, our results may suggest some type of motor specialization (tool use?) in chimpanzees, but indicate no structural asymmetry in terms of relative white to grey matter volume in the posterior (motor) frontal area.

The prefrontal cortex has also been associated with planning and coordinating actions towards higher-level goals [Asplund et al., 2010; Miller and Cohen, 2001]. Moreover, the prefrontal cortex has been suggested to show functional asymmetry: the left prefrontal cortex is associated with action motivation and goal-pursuit behaviour [Berkman and Lieberman, 2010], while the right prefrontal cortex is suggested to be involved in the detection of behaviourally relevant sensory events, particularly when they are salient and unattended [Corbetta and Shulman, 2002]. Considering our analyses demonstrate that apes, compared to monkeys, indicate prefrontal structural lateralization suggesting left prefrontal specialization, we hypothesize that a fundamental aspect of ape versus monkey 'intelligence' may involve an increased capacity for purposeful action-motivated behaviour towards higher-level goals (i.e. goals beyond the immediacy of direct environmental stimuli). Our result that humans follow this ape trend, but to a degree that significantly deviates from their allometry for relative white to grey matter volume, suggests that the human ability 'to approach negative and avoid positive stimuli if that behaviour serves our ultimate goal' [Berkman and Lieberman, 2010, p. 1970] may be the behavioural result of a more general ape trend of neural left prefrontal specialization.

Prefrontal cortex function has also been indicated to involve other metacognitive abilities such as introspection [Fleming et al., 2010], possibly related to self-awareness. Intriguingly, the chimpanzee yields a right prefrontal specialization similar to the one observed in humans and lying markedly outside the allometric trend of other non-human apes. Together with the general ape left prefrontal specialization, this apparent chimpanzee prefrontal specialization may open up questions as to what extent chimpanzees also possess the neural substrate associated with introspective abilities in humans. Such suggestions align with recent research observing that in both humans and chimpanzees the wakeful resting condition of the brain is characterized by a default mode of brain function (including medial prefrontal cortex) [Rilling et al., 2008], which (at least in humans) is associated with mental selfprojection into the past, the future, or another individual's perspective. Although it is clear that these suggestions do not provide evidence of chimpanzee introspection, they do suggest a chimpanzee specialization in prefrontal 
areas associated with introspection and self-awareness in humans.

Our results also have significant implications for comparative investigations into the neural underpinnings of increased cognitive capacities in primates as a clade. Neocortical hyperscaling (i.e. disproportionate increase of the neocortex relative to the rest of the brain) is traditionally suggested as the primary neural substrate for primate 'intelligence'. However, because neocortex volume comprises between 70 and $80 \%$ of total brain volume in apes and between 60 and $75 \%$ of brain volume in monkeys, scaling trends of more detailed (and thus more functionally specific) substructures of the neocortex may reveal a more detailed picture. Smaers et al. [2010] indicated the posterior neocortex (overall neocortex minus frontal lobe) scales isometrically with rest of brain volume (scaling coefficient of 0.99 ), while the frontal lobe significantly hyperscales (scaling coefficient of 1.19). These results suggest that neocortical hyperscaling is primarily due to frontal lobe hyperscaling (despite the fact that the frontal lobe comprises only around $30-35 \%$ of overall neocortex volume). Here we build on these results by focusing on scaling patterns within the frontal lobe. Current results indicate that scaling coefficients along the posterior (motor) part of the frontal lobe relative to rest of brain volume are not significantly different from unity, while scaling coefficients along the anterior (prefrontal) part of the frontal lobe highly deviate from unity in the left hemisphere (scaling coefficients up to 1.35) but not in the right (table 1). These results strongly suggest that in anthropoid primates the left prefrontal cortex (an area comprising not more than $10 \%$ of overall neocortex volume) is the primary factor in explaining overall neocortical hyperscaling.

Allometric studies of the brain can only be fully interpreted in conjunction with studies investigating the genetic, developmental, microstructural cytoarchitectonic and functional basis of the neural system. It is also clear that inferring specific behavioural implications of crossspecies neural structural variation remains difficult. But by modelling quantitative structural changes in particular brain regions across the branches of the tree of life, allometric studies provide unique insight into neural structural changes along millions of years of evolution. This information can then be used to pose new hypotheses in other fields of brain research. Moreover, considering the extensive evidence for structural/functional associations [Carreiras et al., 2009; DeVoogd et al., 1993; Draganski et al., 2004; Fleming et al., 2010; Scholz et al., 2009; Smith et al., 2010], allometric studies utilize an ob- servable measure to estimate the evolution of the neural basis of behaviours often difficult to observe. We provide an allometric analysis of the brain area associated with behaviours often considered to define human uniqueness (action motivation towards higher-level goals, metacognitive introspective capacities, and language). Our results demonstrate anthropoid prefrontal evolution is characterized by different scaling coefficients in the left versus right hemisphere, suggest that the primary factor underlying the evolution of primate brain architecture is left hemispheric prefrontal hyperscaling, and indicate humans are the extreme of a left prefrontal ape specialization in relative white to grey matter volume.

The observed structural lateralization in primate prefrontal cortex evolution reflects an apparent neural adaptive shift distinguishing the ape from the monkey radiation that may be interpreted as a neural substrate underlying a cognitive grade shift between (great) apes and other primates. A possible limitation of our prefrontal delineation procedure is that frontal motor areas (specifically primary motor and premotor cortex) may occupy a smaller proportion of the cortex in humans than in other primates [Blinkov and Glezer, 1968; Brodmann, 1912; Preuss, 2009]; thus that part of the prefrontal cortex may be included in our measure of the posterior end of the frontal lobe. Because humans are also found to deviate from allometry in terms of relative white to grey matter in the left posterior frontal areas, this is not likely to alter our results. It is clear that future research should look to increase sample sizes in order to investigate the effects of intraspecies variability, explore the phylogenetic resolution at which these trends can be observed (between subspecies?) and delineate more detailed prefrontal areas across many primate species in a continued effort to increase the resolution at which we can model the evolution of the brain and brain-behaviour interactions in primates.

\section{Acknowledgements}

We would like to thank Axel Schleicher and John R. Skoyles for useful comments and discussion and Hartmut Mohlberg for making images with the delineation of the motor cortex available from the Jülich/Düsseldorf brain atlas. For training in phylogenetic comparative methods, we thank the AnthroTree Workshop (supported by NSF BCS-0923791). This work was supported by the EC FP6 HANDTOMOUTH project (contract No. 29065). 


\section{References}

Adolphs R (2009): The social brain: neural basis of social knowledge. Annu Rev Psychol 60: 693-716.

Amunts K, Jancke L, Mohlberg H, Steinmetz H, Zilles K (1998): Interhemispheric asymmetry in the human motor cortex and handedness. Eur J Neurosci 10:15402.

-Amunts K, Jancke L, Mohlberg H, Steinmetz H, Zilles K (2000): Interhemispheric asymmetry of the human motor cortex related to handedness and gender. Neuropsychologia 38:304-312.

-Amunts K, Schleicher A, Burgel U, Mohlberg H, Uylings HBM, Zilles K (1999): Broca's region revisited: cytoarchitecture and intersubject variability. J Comp Neurol 412:319-341.

-Asplund CL, Todd JJ, Snyder AP, Marois R (2010): A central role for the lateral prefrontal cortex in goal-direct and stimulus-driven attention. Nat Neurosci 13:507-514.

Berkman ET, Lieberman MD (2010): Approaching the bad and avoiding the good: lateral prefrontal cortical asymmetry distinguishes between action and valence. J Cogn Neurosci 22:1970-1979.

Blinkov SM, Glezer II (1968): Das Zentralnervensystem in Zahlen und Tabellen. Jena, Fischer.

Brodmann K (1912): Neue Ergebnisse über die vergleichende histologische Lokalisation der Grosshirnrinde mit besonderer Berücksichtigung des Stirnhirns. Anat Anz 41:157-216.

Bush EC, Allman JM (2004): The scaling of frontal cortex in primates and carnivores. Proc Natl Acad Sci USA 101:3962-3966.

-Carreiras M, Seghier ML, Baquero S, Estevez A, Lozano A, Devlin JT, Price JC (2009): An anatomical signature for literacy. Nature 461: 983-986.

Catani M, Forkel S, Thiebaut de Schotten M (2010): Asymmetry of the white matter pathways in the brain; in Hugdahl K, Westerhausen R (eds): The Two Halves of the Brain: Information Processing in the Cerebral Hemispheres. Cambridge, MIT Press.

- Corbetta M, Shulman GL (2002): Control of goal-directed and stimulus-driven attention in the brain. Nat Rev Neurosci 3:201-215.

Dadda M, Cantalupo C, Hopkins WD (2008): An association between handedness and neuroanatomical asymmetries in chimpanzees (Pan troglodytes): evidence from the primary motor cortex. Folia Primatol 79:141-142.

Deacon TW (1997): The Symbolic Species. New York, Norton

-DeVoogd T, Krebs JR, Healy SD, Purvis A (1993): Relations between song repertoire size and the volume of brain nuclei related to song: comparative evolutionary analyses among oscine birds. Proc R Soc B Biol Sci 254:75-82.

-Draganski B, Gaser C, Busch V, Schuierer G, Bogdahn U, May A (2004): Changes in grey matter induced by training. Nature 427:311312 .
Dunbar RIM (1996): Grooming, Gossip and the Evolution of Language. London, Faber \& Faber.

Dunbar RIM, Shultz S (2007): Understanding primate brain evolution. Philos Trans R Soc B Biol Sci 362:649-658.

Falk D, Hildebolt C, Cheverud J, Vannier M, Helmkamp RC, Konigsberg L (1990): Cortical asymmetries in frontal lobes of rhesus monkeys (Macaca mulatta). Brain Res 512: $40-45$.

-Fleming SM, Weil RS, Nagy Z, Dolan RJ, Rees G (2010): Relating introspective accuracy to individual differences in brain structure. Science 329:1541-1543.

-Freckleton RP, Harvey PH, Pagel M (2002): Phylogenetic analysis and comparative data: a test and review of evidence. Am Naturalist 160:712-726.

Gage MJG, Freckleton RP (2003): Relative testis size and sperm morphometry across mammals: no evidence for an association between sperm competition and sperm length. Proc $\mathrm{R}$ Soc Lond Ser B Biol Sci 270:625-632.

-Geyer S, Schleicher A, Zilles K (1999): Areas 3a, $3 \mathrm{~b}$, and 1 of human primary somatosensory cortex 1. Microstructural organization and interindividual variability. Neuroimage 10 : 63-83.

Gray JR, Thompson PM (2004): Neurobiology of intelligence: science and ethics. Nat Rev Neurosci 5:471-482.

Harvey PH, Pagel MD (1991): The Comparative Method in Evolutionary Biology. Oxford, Oxford University Press.

Heilbroner PL, Holloway RL (1989): Anatomical brain asymmetry in monkeys: frontal, temporoparietal, and limbic cortex in Macaca. Am J Phys Anthropol 80:203-211.

Holloway RL (2002): How much larger is the relative volume of area 10 of the prefrontal cortex in humans? Am J Phys Anthropol 118: 399-401.

Hopkins WD (ed) (2007): Evolution of Hemispheric Specialization in Primates. London, Academic Press.

-Hopkins WD, Cantalupo C, Taglialatela J (2007a): Handedness is associated with asymmetries in gyrification of the cerebral cortex of chimpanzees. Cereb Cortex 17: 1750-1756.

Hopkins WD, Dunham L, Cantalupo C, Taglialatela JT (2007b): The relationship between handedness, brain asymmetries and corpus callosum size in chimpanzees (Pan troglodytes). Cereb Cortex 17:1757-1765.

Hopkins WD, Taglialatela JP, Dunham L, Pierre $P$ (2007c): Behavioral and neuroanatomical correlates of white matter asymmetries in chimpanzees (Pan troglodytes). Eur J Neurosci 25:2565-2570.
Hopkins WD, Taglialatela JP, Megeurditchian A, Nir T, Schenker NM, Sherwood CC (2008): Gray matter asymmetries in chimpanzees as revealed by voxel-based morphology. Neuroimage 42:491-497.

-Huber R, van Staaden MJ, Kaufman LS, Liem KF (1997): Microhabitat use, trophic patterns, and the evolution of brain structure in African cichlids. Brain Behav Evol 50:167-182.

Hugdahl K, Westerhausen R (eds) (2010): The Two Halves of the Brain: Information Processing in the Cerebral Hemispheres. Cambridge, MIT Press.

-Iwaniuk AN, Wylie DRW (2007): Neural specialization for hovering in hummingbirds: hypertrophy of the pretectal nucleus lentiformis mesencephali. J Comp Neurol 500: 211-221.

Jerison HJ (1997): Evolution of prefrontal cortex; in Krasnegor NA, Lyon R, Goldman-Rakic PS (eds): Development of the Prefrontal Cortex: Evolution, Neurobiology, and Behavior. Baltimore, Paul H. Brookes, pp 9-26.

Jerison HJ (2007): Evolution of the frontal lobes; in Miller BL, Cummings JL (eds): The Human Frontal Lobes: Functions and Disorders. New York, The Guilford Press.

Johnson-Frey SH (2004): The neural bases of complex tool use in humans. Trends Cogn Sci 8:71-78.

Kaas JH (2006): Evolution of the neocortex. Curr Biol 16:R910-R914.

Lindenfors P (2005): Neocortex evolution in primates: the 'social brain' is for females. Biol Lett 1:407-410.

Lindenfors P, Nunn CL, Barton RA (2007): Primate brain architecture and selection in relation to sex. BMC Biol 5:20.

-Marchant LF, McGrew WC, Eibl-Eibesfeldt I (1995): Is human handedness universal? Ethological analyses from three traditional cultures. Ethology 101:239-258.

Martins EP, Hansen TF (1997): Phylogenies and the comparative method: a general approach to incorporating phylogenetic information into the analysis of interspecific data. Am Naturalist 149:646-667.

Matelli M, Luppino G, Geyer S, Zilles K (2004): Motor cortex; in Paxinos G, Mai JK (eds): The Human Nervous System. Amsterdam, Elsevier Academic Press, pp 975-996.

McBride T, Arnold SE, Gur RC (1999): A comparative volumetric analysis of the prefrontal cortex in human and baboon MRI. Brain Behav Evol 54:159-166.

Miller EK, Cohen JD (2001): An integrative theory of prefrontal cortex function. Annu Rev Neurosci 24:167-202.

Pagel M (1999): Inferring the historical patterns of biological evolution. Nature 401:877-884.

-Paradis E, Claude J, Strimmer K (2004): APE: analyses of phylogenetics and evolution in $\mathrm{R}$ language. Bioinformatics 20:289-290. 
Passingham RE (1973): Anatomical differences between the neocortex of man and other primates. Brain Behav Evol 7:337-359.

Passingham RE (2002): The frontal cortex: does size matter? Nat Neurosci 5:190-192.

-Petrides M (2005): Lateral prefrontal cortex: architectonic and functional organization. Philos Trans R Soc B Biol Sci 360:781-795.

- Petrides M, Cadoret GV, Mackey S (2005): Orofacial somatomotor responses in the macaque monkey homologue of Broca's area. Nature 435:1235-1238.

Preuss TM (2009): What is it like to be a human?; in Gazzaniga MS (ed): The Cognitive Neurosciences. Cambridge, MIT Press, pp 49-64.

R Development Core Team (2010): R: A Language and Environment for Statistical Computing, Vienna.

Rilling JK, Barks SK, Parr LA, Preuss TM, Faber TL, Pagnoni G, Bremner JD, Votaw JR (2008): A comparison of resting-state brain activity in humans and chimpanzees. Proc Natl Acad Sci USA 104:17146-17151.

Sakai T, Hirai D, Mikami A, Suzuki J, Hamada Y, Tomonaga M, Tanaka M, Miyabe-Nishiwaki T, Makishima H, Makatsukasa M, Matsuzawa T: Prolonged maturation of prefrontal white matter in chimpanzees. Nat Neurosci, in press.

-Schenker NM, Buxhoeveden DP, Blackmon WL, Amunts K, Zilles K, Semendeferi K (2008): A comparative quantitative analysis of cytoarchitecture and minicolumnar organization in Broca's area in humans and great apes. J Comp Neurol 510:117-128.

-Schenker NM, Desgouttes A-M, Semendeferi K (2005): Neural connectivity and cortical substrates of cognition in hominoids. J Hum Evol 49:547-569.
-Schenker NM, Hopkins WD, Spocter MA, Garrison AR, Stimpson CD, Erwin JM, Hof PR, Sherwood CC (2010): Broca's area homologue in chimpanzees (Pan trogolodytes): probabilistic mapping, asymmetry, and comparison to humans. Cereb Cortex 20: 730-742.

-Schoenemann PT, Sheehan MJ, Glotzer LD (2005): Prefrontal white matter volume is disproportionately larger in humans than in other primates. Nat Neurosci 8:242-252.

Scholz J, Klein MC, Behrens TEJ, Johansen-Berg $\mathrm{H}$ (2009): Training induces changes in whitematter architecture. Nat Neurosci 12:13701371.

Semendeferi K, Armstrong E, Schleicher A, Zilles K, Van Hoesen GW (2001): Prefrontal cortex in humans and apes: a comparative study of area 10. Am J Phys Anthropol 114: 224-241.

Semendeferi K, Lu A, Schenker N, Damasio H (2002): Humans and great apes share a large frontal cortex. Nat Neurosci 5:272-276.

Sherwood CC, Broadfield DC, Holloway RL, Gannon PL, Hof PR (2003): Variability of Broca's area homologue in African great apes: implications for language evolution. Anat Rec A Discov Mol Cell Evol Biol 271:276-285.

Sherwood CC, Holloway RL, Erwin JM, Schleicher A, Zilles K, Hof PR (2004): Cortical orofacial motor representation in old world monkeys, great apes, and humans. I. Quantitative analysis of cytoarchitecture. Brain Behav Evol 63:61-81.

Sherwood CC, Holloway RL, Semendeferi K, Hof PR (2005): Is prefrontal white matter enlargement a human evolutionary specialization? Nat Neurosci 8:537-538.
Smaers JB, Schleicher A, Zilles K, Vinicius L (2010): Frontal white matter volume is associated with brain enlargement and higher structural connectivity in haplorrhine primates. PLoS One 5:e9123.

Smith AR, Seid MA, Jimenez LC, Wcislo WT (2010): Socially induced brain development in a facultatively eusocial sweat bee Megalopta genalis (Halictidae). Proc R Soc B Biol Sci 277:2157-2163.

Smith RJ, Cheverud JM (2002): Scaling of sexual dimorphism in body mass: a phylogenetic analysis of Rensch's rule in primates. Int J Primatol 23:1095-1135.

- Taglialatela JP, Cantalupo C, Hopkins WD (2006): Gesture handedness predicts asymmetry in the chimpanzee inferior frontal gyrus. Neuroreport 7:923-927.

Taglialatela JP, Russell J, Schaeffer JA, Hopkins WD (2008): Communicative signal activates 'Broca's' homologue in chimpanzees. Curr Biol 18:343-348.

-Uylings HBM, Van Eden CG (1990): Qualitative and quantitative comparison of the prefrontal cortex in rat and in primates, including humans. Prog Brain Res 85:31-62.

Uylings HBM, Jacobsen AM, Zilles K, Amunts K (2006): Left-right asymmetry in volume and number of neurons in adult Broca's area. Cortex 42:652-658.

Zhang K, Sejnowski TJ (2000): A universal scaling law between gray matter and white matter of cerebral cortex. Proc Natl Acad Sci USA 97:5621-5626.

Zilles K, Dabringhaus A, Geyer S, Amunts K, Qu M, Schleicher A, Gilissen E, Schlaug G, Steinmetz H (1996): Structural asymmetries in the human forebrain and the forebrain of non-human primates and rats. Neurosci Biobehav Rev 20:593-605. 